\title{
The Impact of the Posterior Parietal and Dorsolateral Prefrontal Cortices on the Optimization of Long-Term versus Immediate Value
}

\author{
Brian G. Essex, ${ }^{1}$ Sarah A. Clinton, ${ }^{1}$ Lucas R. Wonderley, ${ }^{1}$ and David H. Zald ${ }^{1,2}$ \\ ${ }^{1}$ Department of Psychology, Vanderbilt University, Nashville, Tennessee 37240, and 2Department of Psychiatry, Vanderbilt University, Nashville, \\ Tennessee 37240
}

\begin{abstract}
fMRI research suggests that both the posterior parietal cortex (PPC) and dorsolateral prefrontal cortex (DLPFC) help individuals select better long-term monetary gains during intertemporal choice. Previous neuromodulation research has demonstrated that disruption of the DLPFC interferes with this ability. However, it is unclear whether the PPC performs a similarly important function during intertemporal choice, and whether the functions performed by either region impact choices involving losses. In the current study, we used low-frequency repetitive transcranial magnetic stimulation to examine whether the PPC and DLPFC both normally facilitate selection of gains and losses with better long-term value than alternatives during intertemporal choice. We found that disruption of either region in the right hemisphere led to greater selection of both gains and losses that had better immediate, but worse long-term value than alternatives. This indicates that activity in both regions helps individuals optimize long-term value relative to immediate value in general, rather than being specific to choices involving gains. However, there were slightly different patterns of effects following disruption of the right PPC and right DLPFC, suggesting that each region may perform somewhat different functions that help optimize choice.
\end{abstract}

\section{Introduction}

Individuals tend to discount the value of delayed monetary incentives relative to immediate ones and often choose options that have better immediate, but worse long-term value than alternatives (Frederick et al., 2004). While recent research has shown that the lateral prefrontal cortex (PFC) has an important role in helping individuals overcome this bias (Figner et al., 2010), it is unknown whether other brain regions modulate intertemporal choice behavior in a similar way. The posterior parietal cortex (PPC) could play such a role as a number of fMRI studies have shown similar patterns of activation in the PPC and lateral PFC during intertemporal choice (McClure et al., 2004, 2007; Ballard and Knutson, 2009; Bickel et al., 2009; Xu et al., 2009). Consistent with this proposal, fMRI findings show greater activation in the PPC when individuals choose larger delayed rewards over smaller immediate alternatives (Wittmann et al., 2007). Similarly, McClure et al. (2004) report that greater combined activation in a set of regions that includes the lateral PFC and PPC than in a set of other regions sensitive to immediate rewards predicts choice of

\footnotetext{
Received Dec. 9, 2011; revised Aug. 3, 2012; accepted Sept. 4, 2012.

Author contributions: B.G.E. and D.H.Z. designed research; B.G.E., S.A.C., and L.R.W. performed research; B.G.E. analyzed data; B.G.E. and D.H.Z. wrote the paper.

This work was supported by a doctoral dissertation improvement grant from the National Science Foundation to

B.G.E. We thank Martin Gallagher, Warren Lambert, Rebecca Qian, and Tawny Spinelli for their help on this project. The authors declare no competing financial interests.

Correspondence should be addressed to David H. Zald, Department of Psychology, Vanderbilt University, PMB 407817, 2301 Vanderbilt Place, Nashville, TN 37240-7817. E-mail: david.zald@vanderbilt.edu.

DOI:10.1523/JNEUROSCI.6106-11.2012

Copyright $\odot 2012$ the authors $\quad 0270-6474 / 12 / 3215403-11 \$ 15.00 / 0$
}

larger delayed rewards. Yet no studies to date have examined the effects of PPC disruption on intertemporal choice.

In choices involving losses, long-term value is optimized by selecting smaller immediate losses over larger delayed losses. At present, it is unclear whether the PPC and lateral PFC exert similar influences on intertemporal choices involving losses and gains. On the one hand, the neural circuitry that helps individuals decide what rewards to approach may be different from that which helps them decide what aversive outcomes to avoid, an idea consistent with several theories of motivation (Gray, 1981; Panksepp et al., 2002). However, fMRI research indicates that the PPC and lateral PFC are similarly activated during intertemporal choices involving both monetary gains and losses (Bickel et al., 2009; Xu et al., 2009), suggesting that both regions perform similar functions in both types of choices.

To examine whether the PPC facilitates making choices for options with better long-term but worse immediate value than alternatives, we used repetitive transcranial magnetic stimulation (rTMS) to determine the effects of suppressing functioning in the PPC on an intertemporal choice task in which stimuli were tailored to subjects' indifference points. We predicted that disruption of the PPC would lead to greater selection of gain and loss options that had better immediate but worse long-term value than alternatives; disruption was predicted to increase selection of immediate gains and decrease selection of immediate losses. Additionally, we administered a numerical comparison task to verify that any changes in choice were not attributable to alterations in numerical processing given the role of the PPC in mathematical reasoning (Dehaene et al., 2003; Andres et al., 2005; Cappelletti et al., 2007). We also investigated how choices 
changed following disruption of the dorsolateral PFC (DLPFC), to verify past findings that disruption increases selection of immediate gains (Figner et al., 2010), and to see whether it also decreases selection of immediate losses.

\section{Materials and Methods}

Participants. Sixty-four right-handed individuals ( $43.75 \%$ female) between the ages of 18 and 30 (mean age, 21.06; SD, 2.79) from Vanderbilt University and the Nashville community completed this study. All of these participants reported having no history of neurological or psychiatric problems, no females were currently pregnant, and no participants had previously received TMS. All participants completed written informed consent approved by the Vanderbilt University Institutional Review Board. Ninety-five additional subjects were consented but excluded from further study or withdrawn by the investigator $(N=$ $83)$ or chose to withdraw $(N=12)$. Participants were only eligible to complete the rTMS phase of the study if at baseline they showed: (1) evidence of consistent discounting preferences, (2) evidence of delay discounting for both gains and losses, and (3) a level of discounting that allowed generation of the full range of stimuli for rTMS/sham sessions. These requirements limit the generalizability of the conclusions to individuals who possess a moderate amount of delay discounting of both gains and losses, but were necessary to optimally test the core hypotheses of the study. Importantly, the requirement that subjects show delay discounting for both gains and losses was necessary to be able to draw conclusions about relative effects of rTMS on both gains and losses. Methods for determining each of these requirements are described under Session 1 procedures.

Participants were excluded or withdrawn by the investigator due to inconsistent preferences as revealed in Session $1(N=13)$, no delay discounting of losses as revealed on prescreen following consent $(N=$ 15) (initially this prescreen was done following consent, but was later moved before consent due to the high rate of exclusions), risk factors that could increase the chances of having negative effects from TMS (e.g., neurological conditions) $(N=4)$, excessive movement during TMS that made it impossible to reposition the coil within $10 \mathrm{~s}$ following movement onset $(N=2)$, misunderstanding of the task instructions $(N=1)$, experimenter error $(N=2)$, and baseline time preferences revealed in Session 1 that did not allow us to make task stimuli for later sessions $(N=$ 44; further details regarding these excluded individuals given under Session 1 procedures).

Two additional subjects were excluded from analysis because they exhibited choice preferences that were extremely unstable across sessions on the intertemporal choice task (mean proportion of chosen delayed gains or losses changed by $>75 \%$ across Sessions 2 and 3 ). Both of these subjects were in the sham stimulation group and had across-session differences in the mean proportion of chosen delayed gains or losses that were $>4$ SDs from the mean across-session difference score across all subjects. No other participants in the sham or real stimulation groups exhibited changes in preference across sessions of this magnitude, and for all other subjects the across-session difference scores were $<3$ SDs from the overall mean.

Monetary loss prescreen. We screened potential participants before consent with a questionnaire to ensure that we only recruited participants who discounted delayed monetary losses. The screening questionnaire included four questions, each which required participants to write the magnitude of a hypothetical monetary loss to be incurred in 4 weeks that they valued equally to an immediate hypothetical monetary loss of a specific magnitude. Screening was essential because prior research shows that some individuals actually prefer to suffer a monetary loss (or expe- rience other aversive events) sooner rather than later (Frederick et al., 2004). As it is not clear whether individuals who prefer immediate losses over similar (or smaller) later losses are using delay discounting in their decision making, we excluded such individuals from further study. This was necessary because disrupting a brain region that facilitates selecting better long-term options may have different effects in individuals who do not use delay discounting to begin with.

Session 1-indifference point procedure. Following consent, we determined subject indifference points between immediate and delayed monetary rewards. This was done so that we could create choice pairs to be used in later sessions that were matched to each subject's subjective values. We determined immediate equivalents (i.e., dollar amount of an immediate monetary reward that a person values equally to a delayed monetary reward) for four different delayed monetary gains: $\$ 2.50$, $\$ 5.00, \$ 7.50$, and $\$ 10.00$ available at 4 weeks in the future. Determination of immediate equivalents proceeded in ascending order from low to high magnitudes. Subjects were told that they would receive the amount of money associated with one of their choices at the time associated with their selection, and that the trial for which they would receive the money would be selected at random after they completed the task.

For the timing of one trial of this task and presentation of items, see Figure $1 A$. To determine each immediate equivalent, participants were presented with an initial trial in which they made a choice between a delayed monetary gain and an immediate gain of one-half the magnitude of the delayed option. If participants chose the delayed option, the magnitude of the immediate option increased by one-half, and if they chose the immediate option it decreased by one-half. On the next trial, the magnitude of the immediate option changed in a similar way as on the previous trial but only by one-quarter of the original value. Over six trials, the magnitude of the immediate option increased or decreased by progressively smaller amounts [i.e., by $1 /\left(2^{\wedge} x\right)$, where $x$ was trial number] depending on participant responses so that the subjective value of the immediate amount would iteratively approach that of the delayed amount. After the sixth trial, a final catch trial was presented in which the magnitude of the immediate option was higher than the just-calculated immediate equivalent. This provided a check to ensure that subjects were answering according to their preferences (i.e., were answering consistently). If they did not choose the immediate option on the catch trial, the immediate equivalent for that specific delayed monetary amount was determined again. After answering consistently, or after completing the indifference point procedure three times, participants then performed the indifference point procedure for the next delayed monetary amount. If participants answered inconsistently for three indifference point pro- 
Table 1. Immediate values for different trials in the Intertemporal Choice Task

\begin{tabular}{|c|c|c|}
\hline Immediate Relative Value & Total trials & Description \\
\hline Immediate Subjective Value lower than Delayed Subjective Value ${ }^{a}$ & 32 & $\begin{array}{l}\text { Magnitude of immediate option is below immediate equivalent (i.e., immediate value at indifference } \\
\text { point). There were four different percentage distances below the immediate equivalent ( }-10 \text {, } \\
-20,-35 \text {, and }-50 \%) \text {. Subjects who receive sham stimulation should choose the delayed } \\
\text { option if the choice is for gains and the immediate option if the choice is for losses. }\end{array}$ \\
\hline Immediate Subjective Value equal to Delayed Subjective Value & 8 & $\begin{array}{l}\text { Magnitude of immediate option is equal to immediate equivalent (i.e., percentage distance is } 0 \% \text { ). } \\
\text { Neither immediate nor delayed choice is predicted for sham group. }\end{array}$ \\
\hline Immediate Subjective Value higher than Delayed Subjective Value & 32 & $\begin{array}{l}\text { Magnitude of immediate option is above immediate equivalent. There were four different percentage } \\
\text { distances above the immediate equivalent }(10,20,35 \text {, and } 50 \%) \text {. Subjects who receive sham } \\
\text { stimulation should choose the immediate option if the choice is for gains and the delayed option if } \\
\text { the choice is for losses. }\end{array}$ \\
\hline
\end{tabular}

Each delayed gain and each delayed loss magnitude was shown nine times. Each delayed option was paired once with an immediate option located at each of the nine percentage distances from the immediate equivalent of the delayed option. Values at negative distances were taken as a function of the percentage difference between the immediate equivalent and zero. For example, a $-35 \%$ distance indicated that the magnitude of the immediate option was $35 \%$ less than the immediate equivalent. Values at positive distances were calculated as a function of the percentage difference between the immediate equivalent and the delayed option. For example, a $35 \%$ distance indicated that the magnitude of the immediate option was greater than the immediate equivalent by $35 \%$ of the distance between the immediate equivalent and the magnitude of the delayed option.

${ }^{a}$ For both monetary gains and losses, immediate relative value positively scaled with magnitude.

cedures in a row for a given delayed monetary amount, they were excluded. Subjects with inconsistent preferences were excluded due to the potential for high variability across sessions.

Following the indifference point procedure for delayed monetary gains, participants performed the same indifference point procedure for delayed monetary losses. For the loss procedure, the same magnitudes of delayed incentives were used as were used for the gains task, except now subjects chose which of the two values they preferred to lose. They were told that they would have to pay the amount of money for one random choice at the time associated with that choice, which would be selected after they completed the task.

Subjects were excluded if any immediate equivalent was $<10$ cents or if the difference between any immediate equivalent and the magnitude of the associated delayed option was $<10$ cents. This was done to ensure that there would be an adequate range of values above and below each subject's immediate equivalents to create a full range of choice stimuli for further sessions and allowed for consistency of design across subjects. Forty-four individuals were excluded because their immediate equivalents did not fall within this predefined range. Of these subjects, 13 showed too shallow discounting for both gains and losses, 1 showed too shallow discounting for losses and too steep discounting for gains, 26 showed too shallow discounting for losses only, 2 showed too shallow discounting for gains only, 1 showed too steep discounting for losses only, and 1 showed too steep discounting for gains only.

At the end of Session 1, one random trial of intertemporal choice for gain and one random trial of intertemporal choice for loss were selected for payout. Subjects then either received or paid (depending on the trial type) the amount of money at the specified time associated with their choice on that trial.

Sessions 2 and 3. Participants that met eligibility requirements after Session 1 were equally divided into four groups of 16 subjects. Group 1 received real rTMS to the left DLPFC in one session and to the left PPC in a separate session. Group 2 received real rTMS to the right DLPFC in one session and to the right PPC in a separate session. Group 3 received sham rTMS to the same regions as group 1, while group 4 received sham rTMS to the same regions as group 2. Each of the four groups had the same gender distribution (nine males and seven females). Except for the different brain regions stimulated across sessions, procedures in Sessions 2 and 3 were identical.

Before receiving rTMS, participants performed shortened practice versions of the tasks, to make sure they understood the directions. Subjects were told that one gain choice and one loss choice from all of the task trials performed after stimulation would be randomly selected for payment at the end of each session. After completing the practice tasks, participants received real or sham rTMS for $30 \mathrm{~min}$ to either the DLPFC or PPC (details outlined below; see TMS methods).

Intertemporal choice task. Immediately following completion of stimulation, participants completed a 72-trial intertemporal choice task on a computer. On each trial, participants made an intertemporal choice for monetary gain or loss (randomly mixed) followed by a numerical com- parison (Fig. 1). One-half of the choices involved gains and one-half involved losses. Including both gains and losses allowed us to see whether the effects of stimulating each region produced a general impact on discounting or a specific impact limited to the discounting of gains.

The magnitudes and the time of receiving (or paying) the delayed options were the same as in Session $1(\$ 2.50, \$ 5.00, \$ 7.50$, and $\$ 10.00$ to receive or pay in 4 weeks). The magnitude of each immediate option was calculated as a specific percentage difference from that subject's Session 1 immediate equivalent for the delayed option. This allowed us to vary the subjective value of each immediate option (as revealed in Session 1) relative to the associated delayed option, which let us predict the option individuals who received sham stimulation should choose on each trial (Table 1). Previous rTMS research investigating intertemporal choice has not matched stimuli for subjective value across subjects; doing so should increase power to find effects of stimulation since it allows us to measure effects from a common behavioral baseline. The trials administered to each subject in Sessions 2 and 3 were identical, but the order of presentation was randomly determined within each session. Identical trials were given in each session to ensure that changes in choice following rTMS across sessions were not due to differences in the trials administered. However, we note that this design did add a potential confound, in that subjects' responses in Session 3 could have been influenced by their responses in Session 2. However, since the stimulation order was counterbalanced, and Session Number was included as a control variable in analyses, it seems unlikely that the primary findings are related to the repetition of trials across sessions.

Following each intertemporal choice, individuals were asked to make a numerical comparison of the two monetary values that were available on the previous intertemporal choice. This was done to examine whether subjects who received real rTMS had deficits in the ability to compare two monetary values. On one-half of the trials (determined randomly), participants indicated which of the two values was larger and on the other one-half of trials indicated which of the two values was smaller.

At the end of Sessions 2 and 3, one random trial of intertemporal choice for gain and one random trial of intertemporal choice for loss were selected for payout. Subjects then either received or paid (depending on the trial type) the amount of money at the specified time associated with their choice on that trial.

TMS methods. Low-frequency (1 Hz) rTMS was delivered with a Magstim TMS double $70 \mathrm{~mm}$ (figure-eight) coil (Magstim) at 54\% power; this fixed stimulation intensity has been shown to influence behavior in prior rTMS studies of decision making (Knoch et al., 2006b; Figner et al., 2010). The rTMS parameters used were within currently recommended guidelines (Rossi et al., 2009), and stimulation with these parameters leads to suppression of excitability in the targeted region for a period of time following stimulation (Robertson et al., 2003). Sham stimulation was delivered with a Magstim placebo coil, which produced clicks that resembled the sound of rTMS, but without a magnetic pulse. Subjects were blind to the type of stimulation they received. Given the potential for subjects to identify whether they received sham or real stimulation, 
subjects only received one of these types of stimulation. This betweensubjects approach is similar to what has been used in prior rTMS research (Knoch et al., 2006a,b; Camus et al., 2009).

Positioning of the TMS coil was accomplished by using the 10-20 EEG System, which has previously been used to deliver TMS to the DLPFC and PPC with reasonable structural accuracy (Herwig et al., 2003). Others have used the 10-20 EEG system to target the DLPFC with rTMS in a study of intertemporal choice (Figner et al., 2010), or to target the DLPFC or PPC with rTMS in studies of other mental processes (Gerloff et al., 1997; Fierro et al., 2000; Kessels et al., 2000; Hilgetag et al., 2001; Sack et al., 2002; Koch et al., 2005; Schutter and van Honk, 2006). To localize the DLPFC (BA 9/46), the center of the coil was held tangentially to the participant's head with the handle pointing caudally, and placed $1 \mathrm{~cm}$ anterolateral to $\mathrm{F} 3 / \mathrm{F} 4$, which has been suggested to provide better coverage over BA 9/46 than do the points F3/F4 (Herwig et al., 2003). To localize the intraparietal sulcus of the PPC, the center of the coil was held tangentially to the participant's head with the handle pointing rostrally and placed over P3/P4, which has been shown to lie over this area (Sack et al., 2002; Herwig et al., 2003). These specific points for stimulation were marked on a Lycra swim cap that subjects wore; the position of the swim cap was placed in a consistent position on the head across subjects, by using the nasion, inion, and preauricular points as physical landmarks for placement. During stimulation, all participants wore earplugs as protection against the noise of the rTMS pulse. Participants maintained their head position during rTMS administration using both a head rest positioned on the opposite side of the head as the coil and a chin rest, and were visually monitored to ensure that no movement had occurred. In cases in which a participant moved their head, the coil was immediately repositioned over the target. For only two subjects were we unable to reposition the coil within $10 \mathrm{~s}$ following movement onset; this occurred multiple times for these subjects, and because of the inability to quickly reposition the coil, these two subjects were excluded.

All individuals received either real or sham rTMS to the DLPFC or PPC in Session 2 for $30 \mathrm{~min}$ and the same type of stimulation to the other brain region (PPC or DLPFC) on the ipsilateral side for $30 \mathrm{~min}$ in Session 3. The order of stimulating these two regions was counterbalanced across subjects in each group. We used an "off-line" rTMS paradigm; subjects completed tasks after stimulation was completed. Since impairments in behavior following low-frequency rTMS have been shown to last for one-half the time of the previous stimulation (Mottaghy et al., 2002), the tasks following rTMS were limited to the first $15 \mathrm{~min}$ after stimulation.

Statistical analysis methods. To see whether Session 1 preferences were different for subjects who were assigned to each real and sham rTMS group, repeated-measures ANOVAs were performed in PASW Statistics 18 (SPSS). Each repeated-measures ANOVA tested for the effects of Magnitude of the Delayed Option (i.e., $\$ 2.50, \$ 5.00, \$ 7.50, \$ 10.00$ ), Side of Stimulation to be administered in future sessions (i.e., left or right hemisphere), and Stimulation Type to be administered in future sessions (i.e., real or sham rTMS) on mean Session 1 immediate equivalents measured on the Indifference Point Task. Each model contained both main effects and interactions. Two repeated-measures ANOVAs were performed in all: one for immediate equivalents of delayed gains, and one for immediate equivalents of delayed losses.

Using PASW Statistics 18 (SPSS), data from Sessions 2 and 3 were analyzed with Generalized Estimating equations (GEEs), which allow one to model effects while accounting for correlations within observations of individual subjects (Liang and Zeger, 1986). To test the effects of real stimulation relative to sham stimulation, separate GEE models were created for responses following stimulation to each region (i.e., left DLPFC, left PPC, right DLPFC, right PPC). To examine within-subject differences across stimulation region in subjects who received real rTMS, separate GEE models were created for subjects who received stimulation to each hemisphere (i.e., left, right). All models contained an intercept term.

We used GEE models with a logit link function and binomial distribution to predict choice of an immediate option (0, Chose Delayed; 1, Chose Immediate) at the trial level on the Intertemporal Choice Task. Separate models were created to predict choice on gain and loss trials. Independent variables in all models included the factor of Stimulation
Type (0, Sham rTMS; 1, Real rTMS) and the covariates of Immediate Relative Value (i.e., percentage distance of the immediate option from the immediate equivalent of the delayed option, which ranged from -50 to $50 \%$ ) and Session Number (0, Session 2; 1, Session 3), which was included to control for behavior that may have differed across sessions. Additionally, we included the interaction of Immediate Relative Value by Stimulation Type if it was significant $(p<0.05)$. To examine withinsubject differences across region of stimulation in the real rTMS groups, we created similar GEE models, except the within-subject variable Region (0, DLPFC; 1, PPC) was used instead of the between subject variable Stimulation Type; the interaction of Region by Immediate Relative Value was only included if significant.

All other GEE models we created had an identity link function and normal distribution. To predict reaction time at the trial level on the Intertemporal Choice Task (measured in milliseconds), we created separate between-subject GEE models for gain and loss trials. Each model was limited to one region and included the same independent variables as the choice models. Interactions were only included in the final models if significant. We next created between-subject GEE models to predict reaction time on the trial level (measured in milliseconds) on the numerical comparison portion of the Intertemporal Choice Task; models (each limited to one region) contained data from all trials pooled together (i.e., both gain and loss) and were limited to correct trials. Models contained the same independent variables as the models predictive of choice, and an interaction term was only included if significant. We did not create models to examine numerical comparison accuracy, as each real and sham rTMS group performed at near-perfect accuracy on the task [mean accuracy (SD): real left rTMS group, 0.985 (0.015); real right rTMS group, 0.967 (0.049); sham left rTMS group, 0.971 (0.028); sham right rTMS group, $0.981(0.015)]$

We probed for interactions in all GEE models because rTMS could lead to changes in choice or reaction time that depended on the relative values of the two options, in addition to, or rather than, general shifts in behavior. Others have suggested that self-control functions can help individuals choose options with better long-term value when other options have better immediate value (Ballard and Knutson, 2009; Hare et al., 2009; Luo et al., 2009; Figner et al., 2010). Importantly, by including interactions in choice models, it allowed us to examine whether changes in choice supported a role for the PPC and DLPFC in such self-control functions. It would be predicted that a region involved in self-control would show more involvement as the motivational drive to choose an immediate gain (or to avoid choosing an immediate loss) becomes more prepotent, under the assumption that it should be harder to override or inhibit this urge as it increases. Since this urge should become more prepotent as the relative value of the immediate option increases, individuals who receive real rTMS to a region involved in self-control should be increasingly more likely to select the better immediate option than individuals who receive sham stimulation as the relative value of the immediate option increases.

All significant main effects of Stimulation Type or Region and significant interactions involving these variables in GEE models were followed up with post hoc paired comparisons in PASW Statistics 18 to examine whether the effects of rTMS were significantly different across Stimulation Type or Region (depending on analysis). Each paired comparison used a Wald $\chi^{2}$ test to look at differences across Stimulation Type (or Region) in the predicted mean values of a dependent variable from a GEE. For each comparison, the covariate of Session Number was set to the mean value (Session Number, 0.5), and the covariate of Immediate Relative Value was set to one of its nine levels; a separate comparison was done at each of the levels of Immediate Relative Value to see whether significant differences emerged at specific levels of this variable. With the exception of the between-subject choice models depicted in Figures 2 and 3 , the results of post hoc paired comparisons in models without significant interactions are not listed in the text. We note here that in all cases in which there was a significant main effect of Stimulation Type or Region but no significant interaction, all paired comparisons were significant.

For all GEE models, we used unstructured working correlation matrices. However, because the majority of analyses performed on numerical comparison reaction time failed to converge, we also constructed GEE 
Table 2. Mean Session 1 immediate equivalents in the Indifference Point Task

\begin{tabular}{|c|c|c|c|c|c|c|c|c|}
\hline & \multicolumn{2}{|c|}{ Left Real } & \multicolumn{2}{|c|}{ Left Sham } & \multicolumn{2}{|c|}{ Right Real } & \multicolumn{2}{|c|}{ Right Sham } \\
\hline & Mean & (SD) & Mean & (SD) & Mean & (SD) & Mean & (SD) \\
\hline Gain $\$ 2.50$ & 1.226 & $(0.529)$ & 1.146 & $(0.456)$ & 1.424 & $(0.598)$ & 1.510 & $(0.619)$ \\
\hline Gain $\$ 5.00$ & 2.964 & (1.096) & 2.804 & (1.153) & 3.391 & $(0.828)$ & 3.234 & (1.157) \\
\hline Gain $\$ 7.50$ & 4.608 & (1.767) & 4.576 & (1.526) & 4.701 & (1.683) & 4.748 & (1.836) \\
\hline Gain $\$ 10.00$ & 6.836 & $(2.055)$ & 6.194 & $(2.266)$ & 6.523 & (2.274) & 6.681 & $(2.090)$ \\
\hline Loss $\$ 2.50$ & 1.509 & $(0.510)$ & 1.570 & $(0.588)$ & 1.456 & $(0.633)$ & 1.389 & $(0.555)$ \\
\hline Loss $\$ 5.00$ & 3.361 & $(0.988)$ & 3.168 & (1.109) & 3.014 & (1.163) & 2.673 & (1.306) \\
\hline Loss $\$ 7.50$ & 5.119 & (1.523) & 4.640 & (1.699) & 4.843 & (1.769) & 4.916 & (1.587) \\
\hline Loss $\$ 10.00$ & 7.103 & $(2.130)$ & 6.710 & $(2.223)$ & 6.621 & (2.282) & 6.164 & $(2.307)$ \\
\hline
\end{tabular}

Mean Session 1 immediate equivalents for specific magnitudes of delayed gains and losses (listed in left column) available in 4 weeks. Averages are constructed separately for subjects in each of the four rTMS groups (listed in column titles).

Table 3. Mean choice of immediate options in the Intertemporal Choice Task

\begin{tabular}{|c|c|c|c|c|c|c|c|c|}
\hline & \multicolumn{2}{|c|}{ Left DLPFC } & \multicolumn{2}{|c|}{ Left PPC } & \multicolumn{2}{|c|}{ Right DLPFC } & \multicolumn{2}{|c|}{ Right PPC } \\
\hline & Mean & (SD) & Mean & (SD) & Mean & (SD) & Mean & (SD) \\
\hline Real: Gain & 0.389 & $(0.213)$ & 0.389 & $(0.168)$ & 0.511 & $(0.190)$ & 0.474 & $(0.205)$ \\
\hline Sham: Gain & 0.396 & $(0.197)$ & 0.413 & $(0.156)$ & 0.440 & $(0.247)$ & 0.379 & $(0.200)$ \\
\hline Real: Loss & 0.755 & $(0.178)$ & 0.728 & $(0.172)$ & 0.653 & $(0.190)$ & 0.644 & $(0.194)$ \\
\hline Sham: Loss & 0.749 & $(0.183)$ & 0.711 & (0.197) & 0.718 & $(0.176)$ & 0.749 & $(0.245)$ \\
\hline
\end{tabular}

Mean proportion of choices for the immediate option across subjects computed for each type of stimulation (i.e., Real vs Sham) to each side and region. Statistics are collapsed across all gain trials and all loss trials separately.

models for this dependent variable with exchangeable working correlation matrices. To ease comparison of models for each dependent variable, all presented results for a specific dependent variable were based on models with a similar working correlation matrix structure. Thus, while all reported results for numerical comparison reaction time were based on exchangeable matrices, all others were based on unstructured matrices.

\section{Results}

\section{Session 1 indifference points}

Table 2 displays the mean Session 1 immediate equivalents derived from the Indifference Points Task. To ensure that choice preferences were similar across groups before stimulation, we performed a repeated-measures ANOVA on the immediate equivalents measured in Session 1 on the Indifference Point Task. Group assignment differences were tested by looking at the effects of assignment based on Stimulation Type (i.e., real vs sham rTMS) and Side (i.e., left vs right), the interaction between these two variables, and the interactions between these variables and the Magnitude of the Delayed Option. Separate analyses were performed on immediate equivalents for gains and losses. There were no effects of Stimulation Side assignment (for gains: $F_{(1,60)}=$ $0.546, p=0.463$; for losses: $\left.F_{(1,60)}=0.649, p=0.424\right)$ or Stimulation Type assignment (for gains: $F_{(1,60)}=0.096, p=0.757$; for losses: $\left.F_{(1,60)}=0.473, p=0.494\right)$. In addition, there were no significant interactions involving any of the three variables (lowest $p=0.293$; $p$ values are Greenhouse-Geisser corrected). Importantly, these results reveal that before receiving rTMS (i.e., in Session 1), mean levels of delay discounting were similar across all four stimulation groups.

\section{Intertemporal choices}

Effects of real rTMS versus sham

For descriptive statistics of overall mean proportion of choices for immediate gains and losses following each type of stimulation to each side of each brain region, see Table 3. Model parameters for all between-subject GEE models that predict choice of an immediate monetary option are presented in Table 4. All between-subject models contained Stimulation Type, Immediate
Table 4. Between-subject GEE models for Intertemporal Choice

\begin{tabular}{|c|c|c|c|c|c|c|}
\hline & \multicolumn{3}{|l|}{ Gain model } & \multicolumn{3}{|l|}{ Loss model } \\
\hline & B & (SE) & OR & B & (SE) & $O R$ \\
\hline \multicolumn{7}{|l|}{ Between: Left DLPFC } \\
\hline Intercept & $-0.563^{* * *}$ & $(0.155)$ & 0.569 & $0.848^{* * *}$ & $(0.121)$ & 2.335 \\
\hline StimType & 0.138 & $(0.149)$ & 1.148 & 0.162 & $(0.161)$ & 1.176 \\
\hline Imm. Rel. Value & $0.038^{* * *}$ & $(0.003)$ & 1.039 & $-0.027^{* * *}$ & $(0.002)$ & 0.973 \\
\hline Session & 0.194 & $(0.153)$ & 1.215 & $0.588^{* * *}$ & $(0.164)$ & 1.800 \\
\hline \multicolumn{7}{|l|}{ Between: Left PPC } \\
\hline Intercept & $-0.379^{* * *}$ & $(0.102)$ & 0.685 & $1.264^{* * *}$ & $(0.168)$ & 3.538 \\
\hline StimType & $-0.286^{*}$ & $(0.115)$ & 0.751 & 0.065 & $(0.172)$ & 1.067 \\
\hline Imm. Rel. Value & $0.037^{* * *}$ & $(0.003)$ & 1.038 & $-0.032^{* * *}$ & $(0.002)$ & 0.968 \\
\hline Session & -0.040 & $(0.115)$ & 0.961 & $-0.405^{*}$ & $(0.170)$ & 0.667 \\
\hline \multicolumn{7}{|l|}{ Between: Right DLPFC } \\
\hline Intercept & $-0.599^{* * *}$ & $(0.116)$ & 0.549 & $0.943^{* * *}$ & $(0.109)$ & 2.568 \\
\hline StimType & $0.498^{* *}$ & (0.158) & 1.645 & $-0.426^{*}$ & $(0.167)$ & 0.653 \\
\hline Imm. Rel. Value & $0.039^{* * *}$ & $(0.002)$ & 1.039 & $-0.031^{* * *}$ & $(0.002)$ & 0.969 \\
\hline Session & $0.421^{* *}$ & $(0.159)$ & 1.523 & 0.210 & $(0.164)$ & 1.233 \\
\hline \multicolumn{7}{|l|}{ Between: Right PPC } \\
\hline Intercept & -0.177 & $(0.158)$ & 0.838 & $1.307^{* * *}$ & $(0.180)$ & 3.696 \\
\hline StimType & $0.442^{* *}$ & $(0.168)$ & 1.555 & $-0.719^{* * *}$ & $(0.213)$ & 0.487 \\
\hline Imm. Rel. Value & $0.036^{* * *}$ & $(0.003)$ & 1.036 & $-0.022^{* * *}$ & $(0.004)$ & 0.978 \\
\hline Session & $-0.757^{* * *}$ & $(0.171)$ & 0.469 & 0.185 & $(0.200)$ & 1.203 \\
\hline $\begin{array}{l}\text { StimType X Imm. Rel. } \\
\text { Value }\end{array}$ & $0.011^{* *}$ & $(0.004)$ & 1.011 & $-0.011^{*}$ & $(0.005)$ & 0.989 \\
\hline
\end{tabular}

Models predict choice of immediate option on each gain trial (in columns listed "Gain model") or on each loss trial (in columns listed "Loss model"). In the left column, model predictors are listed below each model. Models listed "Between" contain data from subjects who received real rTMS and from subjects who received sham rTMS to region listed after word "Between." OR, Odds ratio $\left(e^{B}\right)$. SE, SE of $B$. Intercept, Model Intercept. Imm. Rel. Value, Immediate Relative Value (percentage distance between the magnitude of the immediate option and the immediate equivalent of the delayed option). StimType, Stimulation Type (0, Sham rTMS; 1 , Real rTMS). Session, Session Number (0, Session 2; 1, Session 3). X, Predictor is interaction of two terms. Models only contained interaction if significant at $p<0.05$.

${ }^{*} p<0.05 ;{ }^{* *} p<0.01 ;{ }^{* * *} p<0.001$

Relative Value, Session Number, and an intercept as predictors. We also included the interaction of Immediate Relative Value by Stimulation Type in all initial models, to assess whether effects of real rTMS on choice depended on the relative value of the immediate reward. However, if this interaction term was not significant ( $p \geq 0.05$ ), we did not include it in the final model.

In all analyses, participants were sensitive to the relative subjective values of the two options as predicted. In all monetary gain models, there was a positive effect of Immediate Relative Value such that subjects were more likely to choose the immediate gain as the relative value of the immediate option increased (all $p<$ $0.001)$. In contrast, in all monetary loss models, there was a negative effect of Immediate Relative Value, such that subjects were less likely to choose the immediate loss as the relative value of the immediate loss increased (all $p<0.001$ ). This reveals that disruption of the DLPFC or PPC did not make participants insensitive to the relative values of the two options and indicates that subjects were attentive to the core study variables in making their choices.

Disruption of the right PPC led to greater choice of options with better immediate, but worse long-term value, and occurred in both the gain and loss domains. Both of these effects were dependent on the relative subjective value of the immediate option, as there was an interaction of Immediate Relative Value by Stimulation Type on choice of both immediate monetary gains $(p=0.009)$ and immediate monetary losses $(p=0.025)$. At low relative values of the immediate option, individuals who received real stimulation chose similarly to those who received sham stimulation. However, as the subjective value of the immediate option increased relative to that of the delayed option, individuals became increasingly more likely to choose immediate monetary gains (Fig. 2A) and increasingly less likely to choose immediate 
monetary losses than individuals who received sham stimulation (Fig. $2 \mathrm{~B}$ ). Across both gain and loss choices, subjects who received real rTMS became increasingly more likely than subjects who received sham rTMS to choose the option with the better immediate, but worse long-term value, as the relative value of the immediate option increased. Notably, this is the effect one would expect following disruption of a self-control function that helps individuals choose an option with better long-term value when another option has better immediate value.

Disruption of the left PPC also affected choice behavior, but only for gain trials (Fig. 2C,D). Real rTMS administered to the left PPC led to fewer choices for immediate monetary gains than did sham stimulation $(p=0.013)$. The effect of left PPC stimulation contrasted with that of right PPC stimulation for gain trials in two ways. First, disruption of the left PPC led to decreased rather than increased choice of monetary gains with better immediate value. Second, the effect occurred across the range of gain trials rather than interacting with Immediate Relative Value.

Like the effects seen following disruption of the right PPC, disruption of the right DLPFC led to greater choice of options with better immediate, but worse long-term value, in both the gain and loss domains. However, unlike the effects seen following disruption of the right PPC, the effects seen following disruption of the right DLPFC were not dependent on the relative subjective value of the immediate option, as similar differences in choice behavior emerged across the range of gain and loss values. Individuals who received real rTMS to the right DLPFC chose more immediate monetary gains $(p=0.002$; Fig. $3 A)$ and less immediate monetary losses $(p=0.011$; Fig. $3 B)$ than did individuals who received sham stimulation to the same region. In contrast, disruption of the left DLPFC did not affect choices (Fig. $3 C, D)$.

Were the effects due to the behavior of the matched sham groups? To corroborate the robustness of our findings and verify that our results were not dependent upon the behavior of the specific matched sham groups, all significant between-group effects were followed up by three additional independent GEE models. Each supplemental model contained identical predictors as the initial models but compared behavior following real rTMS to a region against sham stimulation to one of the three other regions. This allowed us to separately test whether rTMS delivered to a region led to different choice behavior than did sham stimulation to each of the other regions.

Consistent with our primary analyses, real rTMS to the right DLPFC led to greater choice of immediate gains and less choice of immediate losses than did sham stimulation to any of the three other regions (all $p<0.05$ ). Similarly, the effects following real rTMS to the right PPC were consistent with our primary analyses; subjects were more likely to choose immediate gains following real rTMS to the right PPC than following sham stimulation to the right DLPFC or left PPC (both $p<0.05$ ), and there was a
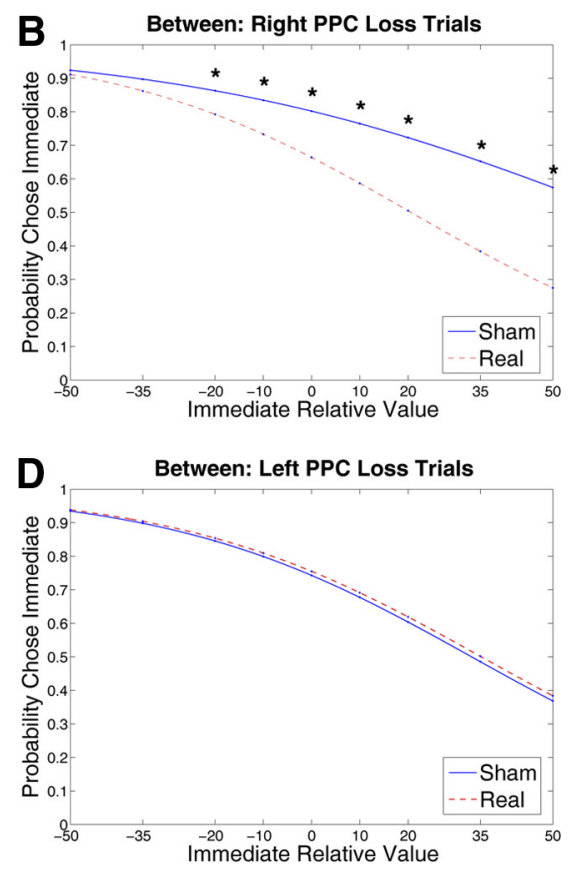

Figure 2. PPC intertemporal choice graphs displaying the results of the between subjects analyses for right PPC gain trials $(\boldsymbol{A})$, in title) between real versus sham stimulation to one side of the PPC (listed in title). Graphs show choice predicted by GEE models. *Paired comparison between stimulation groups using Wald $\chi^{2}$ test significant at $p<0.05$.

trend toward greater choice of immediate gains following real rTMS than following sham to the left DLPFC ( $p=0.056)$. Right PPC stimulation led to less frequent choice of delayed losses in all cases (all $p<0.01$ ). These results stand in contrast to the left PPC, whose effects were only corroborated in one of the three supplemental analyses. Subjects were less likely to choose immediate gains following real rTMS to the left PPC than following sham stimulation to the right DLPFC ( $p=0.01)$ but were not less likely to do so compared with sham stimulation to the left DLPFC or right PPC (both $p>0.05$ ). Because the majority of supplemental analyses addressing the effects of left PPC stimulation did not have significant effects, the left PPC results from the primary analysis must be interpreted with caution.

In the primary analyses, stimulation of the right DLPFC and PPC showed different levels of interaction with Immediate Relative Value, with right PPC stimulation displaying a greater impact as the relative value of the immediate option increased, but no such interaction following right DLPFC stimulation. For gain trials, this distinction was partially supported. As in the initial models, real rTMS to the right DLPFC did not affect the relationship between choice and Immediate Relative Value for gains in any of the supplemental analyses. For the right PPC, two of the three supplemental models contained significant interactions between Immediate Relative Value and Stimulation Type (both $p<$ $0.05)$, and these interactions were in the same direction as those in the primary analysis, with rTMS exerting a greater effect as Immediate Relative Value increased. In contrast, the data did not corroborate a similar difference between the right PPC and right DLPFC for loss trials. None of the supplemental models contrasting the right PPC with other sham sites confirmed an interaction with Immediate Relative Value on loss trials (all $p>0.05$ ). As for the right DLPFC, like in the primary analysis, two of the three 

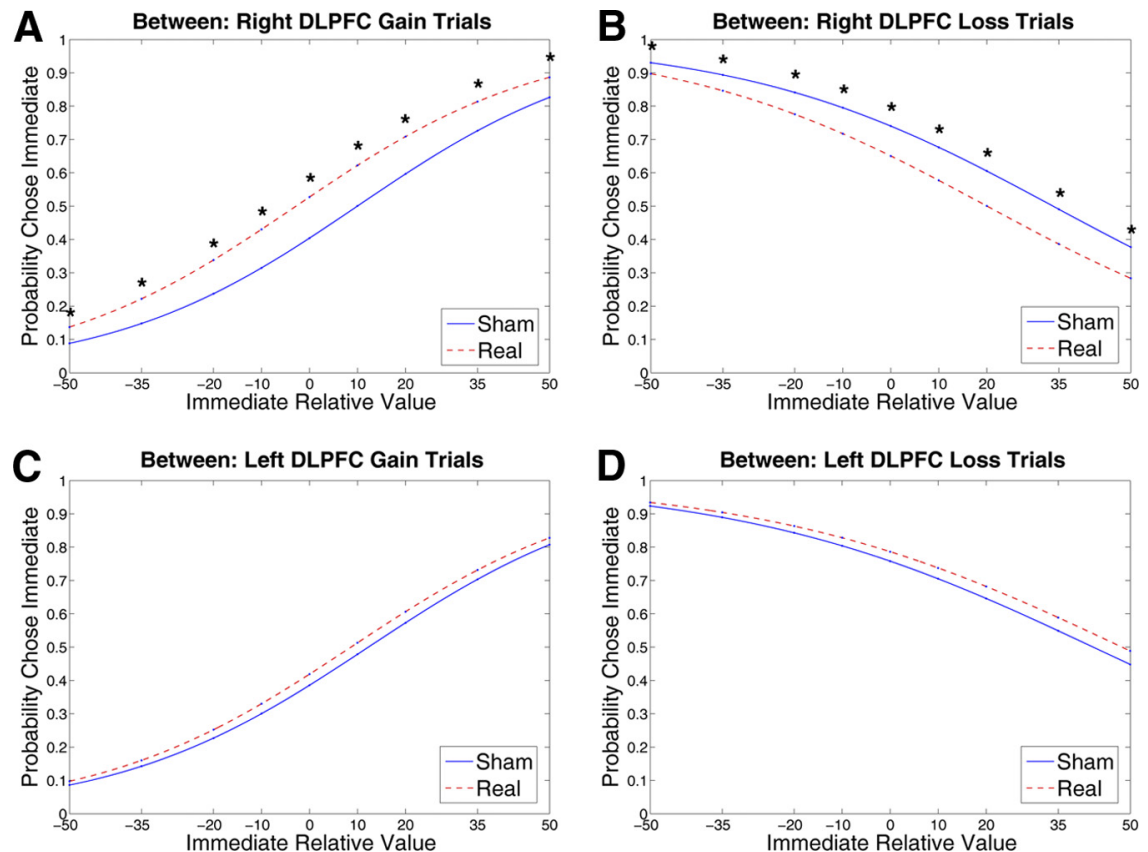

Figure 3. DLPFC intertemporal choice graphs. The graphs show the probability of choosing the immediate option as a function of the relative values of the two options. Numbers on the $x$-axis are the percentage distance between the magnitude of the immediate option and the immediate equivalent of the delayed option (i.e., $x$-axis represents Immediate Relative Value). Each graph compares choice at the average session value (Session $=0.5$ ) on gain or loss trials between subjects receiving real versus sham stimulation to one side of the DLPFC. Graphs show choice predicted by GEE models for the right DLPFC gain trials $(\boldsymbol{A})$, right DLPFC loss trials $(\boldsymbol{B})$, left DLPFC gain trials $(\boldsymbol{C})$, and left DLFC loss trials (D). *Paired comparison between stimulation groups using Wald $\chi^{2}$ test significant at $p<0.05$

Table 5. Within-subject GEE models for Intertemporal Choice

\begin{tabular}{|c|c|c|c|c|c|c|}
\hline & \multicolumn{3}{|l|}{ Gain model } & \multicolumn{3}{|l|}{ Loss model } \\
\hline & $B$ & (SE) & OR & $B$ & (SE) & OR \\
\hline \multicolumn{7}{|c|}{ Real Within: Left } \\
\hline Intercept & $-0.646^{* * *}$ & $(0.081)$ & 0.524 & $1.090^{* * *}$ & $(0.142)$ & 2.974 \\
\hline Region & 0.051 & $(0.073)$ & 1.053 & -0.067 & $(0.064)$ & 0.936 \\
\hline $\begin{array}{c}\text { Imm. Rel. } \\
\text { Value }\end{array}$ & $0.036^{* * *}$ & $(0.003)$ & 1.037 & $-0.030^{* * *}$ & $(0.003)$ & 0.970 \\
\hline Session & 0.070 & $(0.077)$ & 1.072 & $0.178^{* *}$ & $(0.065)$ & 1.195 \\
\hline \multicolumn{7}{|c|}{ Real Within: Right } \\
\hline Intercept & 0.148 & $(0.077)$ & 1.160 & $0.404^{* * *}$ & $(0.099)$ & 1.498 \\
\hline Region & $-0.208^{* * *}$ & $(0.050)$ & 0.812 & 0.052 & $(0.067)$ & 1.054 \\
\hline $\begin{array}{c}\text { Imm. Rel. } \\
\text { Value }\end{array}$ & $0.043^{* * *}$ & $(0.003)$ & 1.044 & $-0.032^{* * *}$ & $(0.002)$ & 0.969 \\
\hline Session & $-0.205^{* * *}$ & $(0.049)$ & 0.815 & $0.249^{* * *}$ & $(0.070)$ & 1.283 \\
\hline
\end{tabular}

Models predict choice of immediate option on each gain trial or on each loss trial. Models listed "Real Within" contain data from subjects who received real rTMS to regions of the hemisphere listed after words "Real Within." Region, Stimulation region (0, DLPFC; 1 , PPC). Other abbreviations are the same as in Table 4. Models only contained interaction if significant at $p<0.05$.

${ }^{*} p<0.05$; ${ }^{* *} p<0.01 ;{ }^{* * *} p<0.001$.

supplemental contrasts with other sham sites showed no evidence of an interaction with Immediate Relative Value (both $p>$ $0.05)$, but a third site did $(p<0.05)$. Thus, it is not possible to confidently conclude that rTMS to the right PPC has a differential impact on choice of losses than does rTMS to the right DLPFC.

Effects of $r$ TMS across region of stimulation

To more thoroughly investigate whether rTMS to the right DLPFC had a different impact on decision making than did rTMS to the right PPC, we examined directly whether the effects of rTMS to each region on choice interacted differently with the relative values of the two options. To do so, we constructed two additional GEE models (one for gain trials and one for loss trials) that contained all gain or loss trials from both sessions 2 and 3 for all subjects who received real or sham rTMS to regions of the right hemisphere. Each model contained the following predictors: Stimulation Type, Region (DLPFC vs PPC), Immediate Relative Value, and all twoway and three-way interactions of these variables. As in other models, Session Number was included as a covariate. Importantly, by including the three-way interaction of Stimulation Type by Region by Immediate Relative Value in these models, we were able to investigate whether there was strong evidence for a differential impact of rTMS to the right DLPFC than to the right $\mathrm{PPC}$ on choice. If this interaction were significant, it would support the findings from the initial between subject analyses showing that rTMS to each of these regions led to different patterns of choice that differed as a function of the relative values of the two options. However, since neither of the models had a significant three-way interaction (both $p>0.05$ ), the results of these supplemental analyses do not provide additional support for a differential effect of rTMS on each region of the right hemisphere.

To determine whether there was a stronger effect of rTMS on the right PPC or right DLPFC, we examined whether there were significant effects of Stimulation Region within subjects who received real rTMS (Table 5). Individuals chose more immediate monetary gains following disruption of the right DLPFC than of the right PPC $(p<0.001)$. However, the magnitude of this effect was small, indicating that choice patterns were not very divergent following disruption of either region. Because of the small size of the effect, it is not discussed further. In contrast to the effect of region of disruption in the right hemisphere on choice for gains, there was no effect of region of disruption in the right hemisphere on choice for losses $(p=0.438)$. Additionally, region of disruption in the left hemisphere did not affect choice of either gains $(p=0.481)$ or losses $(p=0.296)$.

\section{Effects of rTMS across hemisphere}

As a supplemental analysis, we investigated whether subjects who received real rTMS to right hemisphere regions were more likely to choose options with better immediate value than were subjects who received real rTMS to the same regions in the left hemisphere. To assess this, we constructed between-subject GEE models similar to those produced across real and sham groups, except the predictor Stimulation Type was replaced by Hemisphere $(0$, Left; 1, Right). Each model included data from subjects who received real rTMS and was limited to gain or loss choices following stimulation to a specific brain region (PPC or DLPFC). Consistent with the effects of real versus sham stimulation, disruption of either the right PPC or right DLPFC was associated with a greater tendency to choose the option with better immediate value. Individuals who received real rTMS to the right PPC were more likely to choose immediate gains [odds ratio $(\mathrm{OR})=2.064 ; p<0.001$ ] and less likely to choose immediate losses $(\mathrm{OR}=0.636 ; p=0.003)$ than were those 
who received real rTMS to the left PPC. Similarly, those who received real rTMS to the right DLPFC were more likely to choose immediate gains $(\mathrm{OR}=2.137 ; p<0.001)$ and less likely to choose immediate losses $(\mathrm{OR}=0.461 ; p<0.001)$ than were individuals who received real rTMS to the left DLPFC. There were no significant interactions of Immediate Relative Value by Hemisphere in any of the models. The greater tendency to choose better immediate, but worse longterm gains and losses following disruption of right than of left hemisphere regions supports the results of our analyses investigating differences in choice following real versus sham stimulation. Together, both analyses indicate that disruption of either the right PPC or right DLPFC increased choice of gains and losses with better immediate, but worse long-term value than alternatives.

\section{Intertemporal choice reaction time}

We next tested whether reaction time was different across stimulation groups using GEE models. These analyses allowed us to examine whether changes in choice following disruption were associated with changes in the time it took to choose between the options. In particular, we were interested in determining whether biases to select more immediate rewards and less immediate losses were associated with more rapid, impulsive responses. We compared reaction time between real and matched sham rTMS groups following stimulation to regions where there had been a significant main effect of Stimulation Type or interaction including Stimulation Type in the primary between-subject analyses. Predictors in the reaction time models were the same as those in the choice models.

Following rTMS to the right PPC, there was a modest interaction of Stimulation Type by Immediate Relative Value on gain trials $(B=-3.093$; $p=0.014)$; however, paired comparisons revealed that there were no significant differences in reaction time across groups at individual value levels. In loss trials, reaction time was significantly different across groups as reflected in a highly significant main effect of Stimulation Type $(B=329.012 ; p<0.001)$, such that subjects who received real rTMS to the right PPC chose more slowly than sham subjects. Interestingly, the effect on reaction time did not parallel the choice effect for losses, since there was a main effect of Stimulation Type on reaction time, yet an interaction including Stimulation Type on choice.

Following rTMS to the left PPC, there was a significant effect of rTMS on reaction time on gain trials with a significant interaction between Stimulation Type and Immediate Relative Value $(B=2.330 ; p=0.032)$. Post hoc comparisons revealed that subjects who had real rTMS to the left PPC chose more quickly on gain trials than those who had sham rTMS at all relative values of the two options, and differences in reaction time became smaller as Immediate Relative Value increased. There were no significant differences in reaction time across groups following right DLPFC stimulation (Effect of Stimulation Type for gain trials: $B=40.783, p=0.598$; for loss trials: $B=155.188, p=0.094)$.

Together, these data do not provide any support for the idea that modulations producing greater selection of better immediate options are due to more rapid, impulsive decisions. Disruption of the right PPC increased reaction time and led to increased choice of better immediate options on loss trials, while disruption of the left PPC decreased reaction time and led to decreased choice of better immediate options on gain trials. Additionally, the effects of right DLPFC stimulation on choice appeared in the absence of significant effects on reaction time.

\section{Numerical comparisons}

Next, we used GEE models to examine the effects of Stimulation Type, Immediate Relative Value, and Session Number on numerical comparison reaction time for correct trials to see whether disruption of the DLPFC or PPC led to an impaired ability to perform numerical comparisons. We assumed that impairments would be associated with increased reaction times, as others have articulated (Sandrini et al., 2004; Andres et al., 2005; Cappelletti et al., 2007). Each model included Immediate Relative Value (i.e., of the option that had been immediate on the preceding intertemporal choice trial) as an independent variable, because numerical comparisons should become more difficult and slower as the monetary values of the two numbers approach each other, consistent with past research (Moyer and Landauer, 1967). As with other analyses, we only included an interaction term containing Immediate Relative Value if it was significant.

There were no significant differences in reaction time between real and sham groups following stimulation to the right PPC, right DLPFC, or left DLPFC (Effects of Stimulation Type for right PPC: $B=42.651, p=0.545$; for right DLPFC: $B=$ $-17.158, p=0.839$; for left DLPFC: $B=-10.794, p=0.841)$. Thus, there was no evidence that a greater tendency to choose the option with better immediate value following disruption of right hemisphere regions was due to a disrupted ability to perform numerical comparisons. Disruption of only one region, the left PPC, affected numerical comparison performance. Following rTMS delivered to this region, there was an interaction of Immediate Relative Value by Stimulation Type $(B=-1.051 ; p=0.040)$. Despite this interaction, paired comparisons revealed that there were no significant differences in reaction time across groups.

All models revealed that subjects were sensitive to the relative numerical values of the two options. In every GEE model, there was a positive effect of Immediate Relative Value, such that as the value of the immediate option increased and the monetary values of the two options approached each other, reaction time increased (all $p<0.01$ ). This was expected as numerical comparisons should become more difficult as the distance between the two numbers decreases.

As a supplementary analysis, we reran our numerical comparison reaction time models by only modeling reaction time on correct trials in which reaction time was within 2 SDs of each subject's mean reaction time. This was done to remove trials that were outliers and allowed us to better compare our results with those of prior studies that have investigated the effects of TMS or rTMS on numerical comparison abilities, since it is typical to remove outliers before analysis (Sandrini et al., 2004; Andres et al., 2005; Cappelletti et al., 2007). This supplementary analysis led to similar results as the primary reaction time analysis, except there was now no longer any significant interaction of Immediate Relative Value by Stimulation Type following rTMS to the left PPC. Importantly, both the primary and supplementary analyses show that changes in choice were not associated with impairments in the ability to perform numerical comparisons; reaction time on the numerical comparison task was not significantly slower following disruption of any region. 


\section{Discussion}

This study demonstrates that, like the DLPFC, disruption of the PPC alters intertemporal choice. Critically, in this sample of subjects who normally possess delay discounting for both gains and losses, disruption of either the right PPC or right DLPFC led to greater selection of gains and losses with better immediate, but worse long-term value than alternative options. These findings support our hypothesis that the PPC and DLPFC normally facilitate selecting options that optimize long-term value relative to immediate value in general, rather than being specific to gains and the approach motivational system in isolation. Although there may be significant differences in aspects of gain and loss processing and their relationship to approach and avoidance systems (Gray, 1981; Panksepp et al., 2002), our results reveal that the same brain regions exert an influence over decisions involving gains and losses.

Past research suggests that deliberate self-control functions may help individuals choose options with better long-term value when other options have better immediate value (Ballard and Knutson, 2009; Hare et al., 2009; Luo et al., 2009; Figner et al., 2010). Both the PPC (Ballard and Knutson, 2009) and lateral PFC (Ballard and Knutson, 2009; Hare et al., 2009; Figner et al., 2010) have been proposed to play such a role. Disruption of the right PPC produced the pattern of effects that would be expected following disruption of a self-control function. Self-control should be increasingly needed to choose the better long-term option as the relative value of the immediate option increases, and we found that rTMS increasingly biased selection of options with better immediate value as the relative value of the immediate option increased. This suggests that the right PPC may help individuals override, inhibit, or modulate activity that favors selection of better immediate options so that options with better long-term value can be selected. This finding is consistent with the suggestion of Ballard and Knutson (2009) who proposed that the PPC might play a role in controlling responses when there are significant delays based on $\mathrm{AMRI}$ responses in the right PPC that tracked with delay of future rewards and impulsivity. The present study complements these neuroimaging data by demonstrating a causal relationship between right PPC functioning and intertemporal choice.

In considering the changes following disruption of the PPC, it is notable that the results are inconsistent with a model in which the PPC exerts control by preventing hasty decisions. Selfcontrolled decisions might be expected to be slower since it should take more time to make decisions when using deliberative cognitive control processes than when relying on more automatic valuation processes (Stanovich and West, 2000). However, the bias toward better immediate options following right PPC rTMS (and right DLPFC rTMS) was not accompanied by decreased reaction time, and in the loss condition there was evidence of slower responding. Indeed, when shortened reaction times occurred following left PPC rTMS, they were associated with a modest bias toward better long-term options, rather than better immediate options. These results provide little evidence that biases for better immediate options reflected hasty decision making. Similarly, there is no evidence that alterations in choice behavior were due to disruption of numerical comparison processes, as numerical comparison performance was unimpaired following rTMS.

An alternative interpretation of the effects seen following disruption of the PPC is that rTMS directly impacted valuation processes, rather than altering self-control processes. Such a pro- posal would be consistent with prior neurophysiology (Platt and Glimcher, 1999; Dorris and Glimcher, 2004; Sugrue et al., 2004) and fMRI (Peters and Büchel, 2009; Kable and Glimcher, 2010; Prévost et al., 2010) studies showing value-related signals in the parietal cortex. Although other proposals exist (Kable and Glimcher, 2007), McClure et al. (2007) hypothesize that there are two distinct valuation systems that discount the value of delayed rewards as a continuous function of delay: an "impatient" system and a more "patient" system that discounts delayed rewards less steeply than the impatient system. If part of the patient system is disrupted, the impatient system's valuations should dominate the decision process leading to a general shift in preference toward options with better immediate value. Yet disruption of the right PPC did not produce a general shift across all values. Thus, if right PPC involvement in intertemporal choice reflects its role in a patient valuation system, it would appear to primarily exert a measurable influence when immediate values are relatively high. Such a specific change in valuation cannot be confirmed or ruled out in the present study since we examined changes in choice, rather than directly measuring valuation.

Following disruption of the right DLPFC, we saw general shifts in preference toward options with better immediate value, consistent with the patient/impatient model proposed by McClure et al. (2007). This type of observed shift would arise if the DLPFC discounts the value of delayed incentives less steeply than do other brain regions. In contrast, we did not see the types of changes that would be expected following disruption of a classical self-control function, as the modulation of intertemporal choice by rTMS did not scale with immediate relative value. To the extent that the DLPFC is influencing choice via a self-control function, it would have to involve an as-yet-undefined mechanism that exerts a similar influence across the range of relative values.

In considering the possible different contributions of the right PPC and DLPFC to intertemporal choice, it must be noted that, although the data suggest a differential pattern of effects in regards to a self-control hypothesis, the data do not allow for a strong inference regarding dissociable pattern of influence. Supplemental analyses that tested whether right PPC and DLPFC disruption differential interacted with Immediate Relative Value failed to reach statistical significance. Additionally, interactions with Immediate Relative Value did not occur for loss trials in supplemental analyses looking at changes in choice following right PPC disruption. Thus, at present it is not possible to draw strong conclusions about differential effects of right PPC and DLPFC disruption, although the possibility of such differences warrants further study.

The above analysis of the DLPFC data contrasts with that of Figner et al. (2010), who argue that the lateral PFC is involved in self-control rather than valuation during choice, based on their findings that disruption of the left DLPFC with rTMS affected intertemporal choice but not attractiveness ratings of the items that had been used in the choice task. However, the influence of the lateral PFC on valuation may be selective to situations involving choice, with little influence during nonchoice situations, a possibility that cannot be ruled out by the findings of Figner et al. In tasks in which subjects have to make a choice, activations in the DLPFC, or other regions of the lateral PFC, track with some aspect of goal value (Plassmann et al., 2007, 2010; Hare et al., 2008, 2011; Peters and Büchel, 2009; Pine et al., 2009; Kable and Glimcher, 2010). Furthermore, disruption of the right DLPFC with rTMS alters goal values during choice (Camus et al., 2009). 
Future studies are needed to understand the precise involvement or influence on valuation of the DLPFC.

Although both our results and those of Figner et al. (2010) converge on implicating the DLPFC in intertemporal choice, our findings also differ from those of Figner et al. in that we observed altered choice following disruption of the right but not left DLPFC, whereas they only observed altered choice following disruption of the left DLPFC. Our laterality finding is consistent with the finding of Knoch et al. (2006a) that disrupting right but not left DLPFC influences choice behavior, although their study investigated risky rather than intertemporal choice. The precise reasons for the conflicting results across our study and that of Figner et al. are not clear, but a major methodological difference is that we tailored the values to vary systematically around subjects' individual indifference points, whereas Figner et al. did no such individualization. This tailored procedure would be predicted to increase the sensitivity of the task, and could contribute to an enhanced ability to detect right DLPFC modulation, but seems unlikely to account for why we did not replicate the finding by Figner et al. for the left DLPFC. One limitation of our approach to individualizing stimuli is that the procedure of determining indifference points in Session 1 was different from the choice procedure used following real and sham stimulation. It was most likely because of this that stimuli presented at expected indifference points were not consistently selected with $50 \%$ probability even in the sham subjects. However, the differences in procedures across sessions should not have substantially impacted the sensitivity of the tasks. Several other methodological differences arise across studies, including that we used a longer period of rTMS stimulation, had subtle differences in coil positioning, and that Figner et al. used larger magnitude rewards. Critically, unlike Figner et al., our study excluded individuals with very high or very low levels of discounting for both gains and losses. Subsequent studies will be necessary to determine whether sample composition or other methodological differences can explain the laterality differences in DLPFC results.

\section{References}

Andres M, Seron X, Olivier E (2005) Hemispheric lateralization of number comparison. Cogn Brain Res 25:283-290. CrossRef Medline

Ballard K, Knutson B (2009) Dissociable neural representations of future reward magnitude and delay during temporal discounting. Neuroimage 45:143-150. CrossRef Medline

Bickel WK, Pitcock JA, Yi R, Angtuaco EJ (2009) Congruence of BOLD response across intertemporal choice conditions: fictive and real money gains and losses. J Neurosci 29:8839-8846. CrossRef Medline

Camus M, Halelamien N, Plassmann H, Shimojo S, O’Doherty J, Camerer C, Rangel A (2009) Repetitive transcranial magnetic stimulation over the right dorsolateral prefrontal cortex decreases valuations during food choices. Eur J Neurosci 30:1980-1988. CrossRef Medline

Cappelletti M, Barth H, Fregni F, Spelke ES, Pascual-Leone A (2007) rTMS over the intraparietal sulcus disrupts numerosity processing. Exp Brain Res 179:631-642. CrossRef Medline

Dehaene S, Piazza M, Pinel P, Cohen L (2003) Three parietal circuits for number processing. Cogn Neuropsychol 20:487-506. CrossRef Medline

Dorris MC, Glimcher PW (2004) Activity in posterior parietal cortex is correlated with the relative subjective desirability of action. Neuron 44:365378. CrossRef Medline

Fierro B, Brighina F, Oliveri M, Piazza A, La Bua V, Buffa D, Bisiach E (2000) Contralateral neglect induced by right posterior parietal rTMS in healthy subjects. Neuroreport 11:1519-1521. CrossRef Medline

Figner B, Knoch D, Johnson EJ, Krosch AR, Lisanby SH, Fehr E, Weber EU (2010) Lateral prefrontal cortex and self-control in intertemporal choice. Nat Neurosci 13:538-539. CrossRef Medline

Frederick S, Loewenstein G, O’Donoghue T (2004) Time discounting and time preference: a critical review. In: Advances in behavioral economics
(Camerer CF, Loewenstein G, Rabin M, eds), pp 162-222. Princeton: Princeton UP.

Gerloff C, Corwell B, Chen R, Hallett M, Cohen LG (1997) Stimulation over the human supplementary motor area interferes with the organization of future elements in complex motor sequences. Brain 120:1587-1602. CrossRef Medline

Gray JA (1981) A critique of Eysenck's theory of personality. In: A model for personality (Eysenck HJ, ed), pp 246-276. Berlin: Springer.

Hare TA, O’Doherty J, Camerer CF, Schultz W, Rangel A (2008) Dissociating the role of the orbitofrontal cortex and the striatum in the computation of goal values and prediction errors. J Neurosci 28:5623-5630. CrossRef Medline

Hare TA, Camerer CF, Rangel A (2009) Self-control in decision-making involves modulation of the vmPFC valuation system. Science 324: 646-648. CrossRef Medline

Hare TA, Malmaud J, Rangel A (2011) Focusing attention on the health aspects of foods changes value signals in vmPFC and improves dietary choice. J Neurosci 31:11077-11087. CrossRef Medline

Herwig U, Satrapi P, Schönfeldt-Lecuona C (2003) Using the international 10-20 EEG system for positioning of transcranial magnetic stimulation. Brain Topogr 16:95-99. CrossRef Medline

Hilgetag CC, Théoret H, Pascual-Leone A (2001) Enhanced visual spatial attention ipsilateral to rTMS-induced "virtual lesions" of human parietal cortex. Nat Neurosci 4:953-957. CrossRef Medline

Kable JW, Glimcher PW (2007) The neural correlates of subjective value during intertemporal choice. Nat Neurosci 10:1625-1633. CrossRef Medline

Kable JW, Glimcher PW (2010) An "as soon as possible" effect in human intertemporal decision making: behavioral evidence and neural mechanisms. J Neurophysiol 103:2513-2531. CrossRef Medline

Kessels RP, d'Alfonso AA, Postma A, de Haan EH (2000) Spatial working memory performance after high-frequency repetitive transcranial magnetic stimulation of the left and right posterior parietal cortex in humans. Neurosci Lett 287:68-70. CrossRef Medline

Knoch D, Gianotti LR, Pascual-Leone A, Treyer V, Regard M, Hohmann M, Brugger P (2006a) Disruption of right prefrontal cortex by lowfrequency repetitive transcranial magnetic stimulation induces risktaking behavior. J Neurosci 26:6469-6472. CrossRef Medline

Knoch D, Pascual-Leone A, Meyer K, Treyer V, Fehr E (2006b) Diminishing reciprocal fairness by disrupting the right prefrontal cortex. Science 314: 829-832. CrossRef Medline

Koch G, Oliveri M, Torriero S, Carlesimo GA, Turriziani P, Caltagirone C (2005) rTMS evidence of different delay and decision processes in a fronto-parietal neuronal network activated during spatial working memory. Neuroimage 24:34-39. CrossRef Medline

Liang KY, Zeger SL (1986) Longitudinal data-analysis using generalized linear-models. Biometrika 73:13-22. CrossRef

Luo S, Ainslie G, Giragosian L, Monterosso JR (2009) Behavioral and neural evidence of incentive bias for immediate rewards relative to preferencematched delayed rewards. J Neurosci 29:14820-14827. CrossRef Medline

McClure SM, Laibson DI, Loewenstein G, Cohen JD (2004) Separate neural systems value immediate and delayed monetary rewards. Science 306 : 503-507. CrossRef Medline

McClure SM, Ericson KM, Laibson DI, Loewenstein G, Cohen JD (2007) Time discounting for primary rewards. J Neurosci 27:5796-5804. CrossRef Medline

Mottaghy FM, Gangitano M, Sparing R, Krause BJ, Pascual-Leone A (2002) Segregation of areas related to visual working memory in the prefrontal cortex revealed by rTMS. Cereb Cortex 12:369-375. CrossRef Medline

Moyer RS, Landauer TK (1967) Time required for judgements of numerical inequality. Nature 215:1519-1520. CrossRef Medline

Panksepp J, Knutson B, Burgdorf J (2002) The role of brain emotional systems in addictions: a neuro-evolutionary perspective and new "selfreport" animal model. Addiction 97:459-469. CrossRef Medline

Peters J, Büchel C (2009) Overlapping and distinct neural systems code for subjective value during intertemporal and risky decision making. J Neurosci 29:15727-15734. CrossRef Medline

Pine A, Seymour B, Roiser JP, Bossaerts P, Friston KJ, Curran HV, Dolan RJ (2009) Encoding of marginal utility across time in the human brain. J Neurosci 29:9575-9581. CrossRef Medline

Plassmann H, O’Doherty J, Rangel A (2007) Orbitofrontal cortex encodes 
willingness to pay in everyday economic transactions. J Neurosci 27: 9984-9988. CrossRef Medline

Plassmann H, O'Doherty JP, Rangel A (2010) Appetitive and aversive goal values are encoded in the medial orbitofrontal cortex at the time of decision making. J Neurosci 30:10799-10808. CrossRef Medline

Platt ML, Glimcher PW (1999) Neural correlates of decision variables in parietal cortex. Nature 400:233-238. CrossRef Medline

Prévost C, Pessiglione M, Météreau E, Cléry-Melin ML, Dreher JC (2010) Separate valuation subsystems for delay and effort decision costs. J Neurosci 30:14080-14090. CrossRef Medline

Robertson EM, Théoret H, Pascual-Leone A (2003) Studies in cognition: the problems solved and created by transcranial magnetic stimulation. J Cogn Neurosci 15:948-960. CrossRef Medline

Rossi S, Hallett M, Rossini PM, Pascual-Leone A; Safety of TMS Consensus Group (2009) Safety, ethical considerations, and application guidelines for the use of transcranial magnetic stimulation in clinical practice and research. Clin Neurophysiol 120:2008-2039. CrossRef Medline

Sack AT, Sperling JM, Prvulovic D, Formisano E, Goebel R, Di Salle F, Dierks T, Linden DE (2002) Tracking the mind's imagine in the brain. II: Transcranial magnetic stimulation reveals parietal asymmetry in visuospatial imagery. Neuron 35:195-204. CrossRef Medline
Sandrini M, Rossini PM, Miniussi C (2004) The differential involvement of inferior parietal lobule in number comparison: a rTMS study. Neuropsychologia 42:1902-1909. CrossRef Medline

Schutter DJ, van Honk J (2006) Increased positive emotional memory after repetitive transcranial magnetic stimulation over the orbitofrontal cortex. J Psychiatry Neurosci 31:101-104. Medline

Stanovich KE, West RF (2000) Individual differences in reasoning: implications for the rationality debate? Behav Brain Sci 23:645-726. CrossRef Medline

Sugrue LP, Corrado GS, Newsome WT (2004) Matching behavior and the representation of value in the parietal cortex. Science 304:1782-1787. CrossRef Medline

Wittmann M, Leland DS, Paulus MP (2007) Time and decision making: differential contribution of the posterior insular cortex and the striatum during a delay discounting task. Exp Brain Res 179:643-653. CrossRef Medline

Xu L, Liang ZY, Wang K, Li S, Jiang T (2009) Neural mechanism of intertemporal choice: from discounting future gains to future losses. Brain Res 1261:65-74. CrossRef Medline 